\section{Clinical interventions in COPD \\ P150 HOW WELL DO PATIENTS WITH CHRONIC RESPIRATORY FAILURE SECONDARY TO COPD AND THEIR CARERS ADAPT TO LONG-TERM OXYGEN THERAPY AT HOME?}

doi:10.1136/thx.2010.151043.1

C Wrench. Sheffield Teaching Hospitals NHS Foundation Trust, Sheffield, UK

Introduction and objectives Long-term oxygen therapy for the treatment chronic respiratory failure requires a commitment by patients to use $15 \mathrm{~h}$ oxygen per day and yet little is known about the impact of this demanding treatment on daily living. A phenomenological appro/ach was used to explore the personal experiences of patients starting long-term oxygen therapy at home and their carers. All patients had a primary diagnosis of COPD with chronic respiratory failure and had completed formal oxygen assessment in the Oxygen Assessment Clinic. The study was ethically approved.

Methods The research tool was a single, semi-structured recorded interview carried out within the participants' own home within 4 weeks of commencing oxygen. Participants were recruited from clinic over a 2 -month period and consisted of seven patient participants (6 female, 1 male) and four carer participants ( 3 female, 1 male). For the purpose of this study, a carer was defined as someone who had daily contact with the patient. A semi-structured interview was carried out in the participants' own home. Interviews were recorded and then transcribed verbatim. Patients and carers were interviewed separately to ensure confidentiality. Due to the chronic breathlessness of the patient participants, interview times were limited to between 30 and 45 min. Analysis was carried out using Colaizzi's seven stage process (1978).

Results Patient participants described emotions ranging from initial fear, anger, and frustration to final acceptance. This journey was echoed in the experiences of the carers and shared elements of the grieving process (Kubler-Ross). Some patients and carers had begun to develop coping strategies to help with day-to-day activities, such as using ambulatory oxygen to complete simple chores or using breathing exercises to relax.

Conclusions The impact of long-term oxygen therapy on the participants of this study, both emotionally and physically, was considerable. Formal oxygen assessment is undoubtedly essential but patients and their carers need additional educational and emotional support from an experienced Health Care Professional, particularly during the initial stages of their treatment.

\section{P151 REDUCED MORTALITY WITH $\beta$ BLOCKERS WHEN ADDED TO STEPWISE THERAPY FOR COPD}

doi:10.1136/thx.2010.151043.2

${ }^{1} \mathrm{P}$ M Short, ${ }^{2} \mathrm{~S}$ I W Lipworth, ${ }^{3} \mathrm{D}$ H Elder, ${ }^{4} \mathrm{~S}$ Schembri, ${ }^{1} \mathrm{~B} \mathrm{~J}$ Lipworth. ${ }^{1}$ Asthma and Allergy Research Group, University of Dundee, Dundee, UK; ${ }^{2}$ Bute Medical School, University of St. Andrews, St. Andrews, UK; ${ }^{3}$ Department of Cardiovascular Medicine, University of Dundee, Dundee, UK; ${ }^{4}$ Department of Respiratory Medicine, NHS Tayside, Dundee, UK

Introduction and objectives We wished to examine if $\beta$ blockers reduced mortality in addition to stepwise therapy for COPD, independent of cardiovascular disease.

Methods We searched data provided by the Information Services Division of NHS Scotland, to identify patients in NHS Tayside, who required a hospital admission due to COPD. We then searched the NHS Tayside Respiratory Disease Information System (TARDIS) to identify patients since January 2001 to January 2010 who had a documented history of COPD: to collect data on lung function, smoking history and $\mathrm{SaO} 2$. We collected prescription data from the
Tayside Community Prescription database and history of death in our population from the General Register Office. We also collected history of diabetes and admission to hospital due to cardiovascular disease in our cohort. Using Cox Regression Survival analysis, we calculated the hazard ratios for mortality based upon step wise inhaled therapy and $\beta$-blocker use. Cardiovascular and respiratory hospital admissions, diabetes, smoking, age at diagnosis, cardiac drug use, $\mathrm{FEV} 1 \%$ and $\mathrm{SaO} 2$ were also included in our model.

Results 27170 patients were initially identified with a hospital admission due to COPD. 6394 patients were identified through the TARDIS database of which 5977 patients were over 50 years of age and used for analysis. Mean age at diagnosis was 69 years. Mean FEV $1 \%$ was $62 \%$. $89 \%$ of beta-blockers (BB) were cardioselective. All patients were receiving $\mathrm{SABA} \pm$ ipratropium. Adjusted hazard ratios for mortality and 95\% CI's relative to the control group (mean $\mathrm{FEV}_{1}$ $71 \%)$ receiving SABA \pm ipratropium only $(n=915)$ are displayed in the Abstract P151 Table 1.

Abstract P151 Table 1 Adjusted hazard ratios for mortality based on treatment group

\begin{tabular}{lclll}
\hline Intervention & Number & Mean FEV $_{\mathbf{1}}$ (\%) & Adjusted HR & $\mathbf{9 5 \%} \mathbf{~ C I}$ \\
\hline ICS+LABA+Tio+BB & 268 & $55.3 \%$ & 0.32 & $0.24-0.43$ \\
ICS+LABA+Tio & 2082 & $52.5 \%$ & 0.5 & $0.44-0.58$ \\
ICS+LABA+BB & 148 & $67.9 \%$ & 0.47 & $0.33-0.66$ \\
ICS+LABA & 922 & $66.5 \%$ & 0.76 & $0.65-0.88$ \\
ICS+Tio & 170 & $60.1 \%$ & 0.6 & $0.45-0.79$ \\
ICS+BB & 110 & $64.8 \%$ & 0.49 & $0.34-0.71$ \\
ICS & 456 & $69.1 \%$ & 0.79 & $0.65-0.95$ \\
LABA/Tio (no ICS) + BB & 131 & $65.2 \%$ & 0.65 & $0.46-0.91$ \\
LABA/Tio (no ICS) & 502 & $60.6 \%$ & 0.75 & $0.62-0.9$ \\
BB (no ICS) & 273 & $73.6 \%$ & 0.74 & $0.57-0.94$ \\
\hline
\end{tabular}

Conclusion $\beta$ Blockers reduce mortality in an additive fashion to stepwise therapy for COPD, independently of cardiac hospital admissions and medications.

\section{Clinical aspects of NIV

P152
EFFECT OF OBESITY IN PATIENTS ADMITTED TO NON
INVASIVE VENTILATION (NIV) UNIT WITH ACUTE
HYPERCAPNIC RESPIRATORY FAILURE (AHRF)

doi:10.1136/thx.2010.151043.3

${ }^{1} \mathrm{C}$ M Thippanna, ${ }^{1} \mathrm{~A}$ Thomas, ${ }^{1} \mathrm{~W}$ Tosh, ${ }^{2} \mathrm{~B}$ Chakraborty, ${ }^{1} \mathrm{~B}$ Beauchamp, ${ }^{1} \mathrm{D}$ Banerjee, ${ }^{1} \mathrm{R}$ Mukherjee. ${ }^{1}$ Heart of England NHS Foundation Trust, Birmingham, UK; ${ }^{2}$ School of Mathematics, University of Birmingham, Birmingham, UK

Introduction The prevalence of obesity, defined by the World Health Organization as a body mass index (BMI) greater than $30 \mathrm{~kg} / \mathrm{m}^{2}$, is increasing in all developed countries. Obesity as the cause of admission or complicating in-patient stay has been increasing over the last decade. Obesity in intensive care unit (ICU) is significantly related to prolonged duration of mechanical ventilation and length of stay. Obesity is a known risk factor for acute hypercapnic respiratory failure (AHRF).

Methods An observational, single-centre, retrospective analysis of physician diagnosed obese patients requiring NIV for AHRF between April 2005 to March 2006 and April 2009 to March 2010. Demographic data including age, sex, height, weight, date of initiating NIV and length of stay in hospital were collected and BMI was calculated.

Results 154 patients were admitted in the period April 2005 to March 2006 and 160 patients in the period April 2009 to March 2010 to the dedicated NIV unit. The admissions to the unit with 